\title{
SEMI-ANALYTICAL PERIOD-DOUBLING CHATTER ANALYSIS IN THIN WALL MILLING
}

\author{
M. Sanz-Calle ${ }^{1 *}$, J. Munoa ${ }^{1,2}$, A. Iglesias ${ }^{1}$, LN. Lopez de Lacalle², Z. Dombovari3 \\ ${ }^{1}$ IDEKO, Department of Dynamics \& Control, Elgoibar, Spain \\ ${ }^{2}$ University of the Basque Country, Mechanical Engineering Department, Bilbao, Spain \\ ${ }^{3}$ Budapest University of Technology and Economics, MTA-BME Lendulet Machine Tool Vibration Research Group, \\ Budapest, Hungary
}

*Corresponding author; e-mail: msanz@ideko.es

\begin{abstract}
During thin-walled part milling, a dominant flexible direction perpendicular to the feed motion is most likely to exist, which allows to formulate the stability problem in the frequency domain in a very simple form. By these means, the existence of optimal engagements under up-milling strategy for achieving a theoretical infinite Hopf stability have already been demonstrated. However, period-doubling chatter can also pose a limit to the productivity in thin wall milling, but the knowledge on optimal engagements that can cancel this kind of chatter is inexistent.

This paper discusses the effect of the radial engagement and number of flutes on flip stability in a dimensionless way and independent on the system dynamics. Up- and down- milling strategies are compared: a larger period-doubling prevalence is identified in the former, although in terms of absolute critical depth of cut, up-milling outperforms down-milling for most of the practical cases. It is also demonstrated that even though it is possible to find optimal engagements that minimise the flip likelihood, it is impossible to totally cancel the period-doubling chatter by simply tuning the radial engagement, which leaves the cutter helix tuning as the only way to completely eliminate flip chatter. Finally, the obtained results are validated through semidiscretisation simulations.
\end{abstract}

Keywords:

Milling; chatter; period-doubling;

\section{INTRODUCTION}

Regenerative chatter is, together with static deflections, one of the main factors limiting the productivity when milling thin-walled parts. Their very low stiffness and internal damping properties result in a large dynamic flexibility at points to be machined. This highly promotes the occurrence of large amplitude chatter vibrations that can lead to deep surface marks, rapid tool wear and accelerated machine tool component damage.

Regenerative chatter is a type of self-excited vibration caused by the regenerative effect [Tobias 1958, Tlusty 1963], whereby each cutting edge cuts the wavy pattern left in the previous pass. Mathematically, this can be described as an autonomous delay differential equation (DDE), which under certain machining parameters can lead to Hopf-kind of instabilities, causing the well-known machining chatter. In the case of milling, the governing equation becomes a time-periodic DDE due to the parametric excitation induced by the time-periodicity of the so-called directional factor matrix. The time-periodic DDEs not only exhibit Hopf bifurcations, but also period-doubling -flip- instabilities [Davies 2000]. In other heavy-duty milling processes the parametric excitation is negligible and seldom limits the stability of the process. However, in thin wall milling the period-doubling chatter can pose a real problem when searching for chatter-free machining conditions. The low radial immersion and the small number of teeth used can much promote a highly interrupted cutting and, thus, a significant parametric excitation.

Among the different techniques with which chatter can be avoided [Munoa 2016], process planning-based methods backed by stability models constitute a very industrially attractive alternative. These models are usually based on complex time-discrete algorithms such as semidiscretisation [Insperger 2011] or full discretisation [Ding 2010] methods due to the multi-dimensional nature of the limiting modes and in order to handle the highly interrupted cutting. However, like with any other numerical method, the insight of the effect of machining parameters on milling stability is lost, turning the process planning task into a long trial and error process.

The stability of the time-periodic DDEs arising in milling can be alternatively studied by means of the so-called frequency domain methods. Frequency domain methods parametrically provide the root-crossing curves by successively solving an eigenvalue problem. If only the 


\section{HSM \\ 2021 \\ MM Science Journal | www.mmscience.eu \\ ISSN 1803-1269 (Print) | ISSN 1805-0476 (Online) \\ Special Issue | HSM 2021 \\ $16^{\text {th }}$ International Conference on High Speed Machining \\ October 26-27, 2021, Darmstadt, Germany}

DOI: 10.17973/MMSJ.2021_11_2021167

zeroth harmonic of the directional factor is considered, a rapid calculation of the Hopf critical limits is possible, which is commonly known as the zeroth order algorithm (ZOA, [Altintas 1995]). ZOA solution can be extended by considering higher harmonics of the directional factor matrix [Minis 1993], leading to the multifrequency method (MF, [Budak 1998]), able to capture both Hopf and flip instabilities [Merdol 2004, Munoa 2013]. In any case, these general frequency domain algorithms are still far from providing the key relations between the milling parameters and the system stability.

In thin-walled part milling, a dominant modal direction perpendicular to the feed motion is most likely to exist, so the directional factor can be condensed onto a single coefficient. Hence, the stability problem can be reformulated in the frequency domain in a simpler form [Zatarain 2010], where the implications of the mean directional factor and its harmonics on Hopf and flip stability limits can be devised [Iglesias 2016]. By this means, it has already been demonstrated not only that up-milling always outperforms down-milling in terms of Hopf stability, but also that it is possible to find an engagement at which a theoretical infinite Hopf stability can be achieved [SanzCalle, 2021]. When it comes to the optimisation of the period-doubling stability, it is well-known that it is possible to totally cancel the flip chatter by properly tuning the cutter helix pitch and the axial depth of cut [Zatarain 2006, 2010], whereas the influence of radial engagement and number of flutes on period-doubling stability still remains unknown.

Therefore, this paper studies the influence of the radial engagement and number of flutes on the shape, prevalence and critical value of the period-doubling stability limit, independently of the dynamics of the part or other process parameters. To this end, the thin wall milling stability problem is formulated in Section 2. In Section 3, up- and down-milling strategies are compared, and the optimisation of the flip stability with the radial engagement is discussed. Finally, in Section 4 the findings are demonstrated through semidiscretisation calculations.

\section{STABILITY OF INTERRUPTED MILLING OF THIN-WALLED PARTS}

Most parts with thin-walled features are generally machined by successive milling passes perpendicular to their 'thickness direction' $(y)$ along which they mainly exhibit their flexibility. Consequently, the milling dynamics can be well described by means of a unidirectional milling model as shown in Fig. 1.

\subsection{Regenerative unidirectional milling model}

Consider a single $y$-mode with natural frequency $\omega_{\mathrm{n}}$, damping ratio $\zeta$ and modal stiffness $k$ and a cylindrical cutter with $Z$ regularly spaced teeth rotating at a constant spindle speed $\Omega$. Under these considerations, the DDE governing the milling dynamics can be expressed as

$$
\begin{aligned}
\ddot{y}(t)+2 \zeta \omega_{\mathrm{n}} \dot{y}(t)+ & \omega_{\mathrm{n}}^{2} y(t)=\frac{\omega_{\mathrm{n}}^{2}}{k} F(t, y(t), y(t-\tau)) \\
& =\frac{\omega_{\mathrm{n}}^{2}}{k} a K_{t \mathrm{c}} B(t)(y(t-\tau)-y(t)) .
\end{aligned}
$$

where $a$ and $K_{t c}$ stand for the axial depth of cut and tangential cutting coefficient, respectively. The regenerative

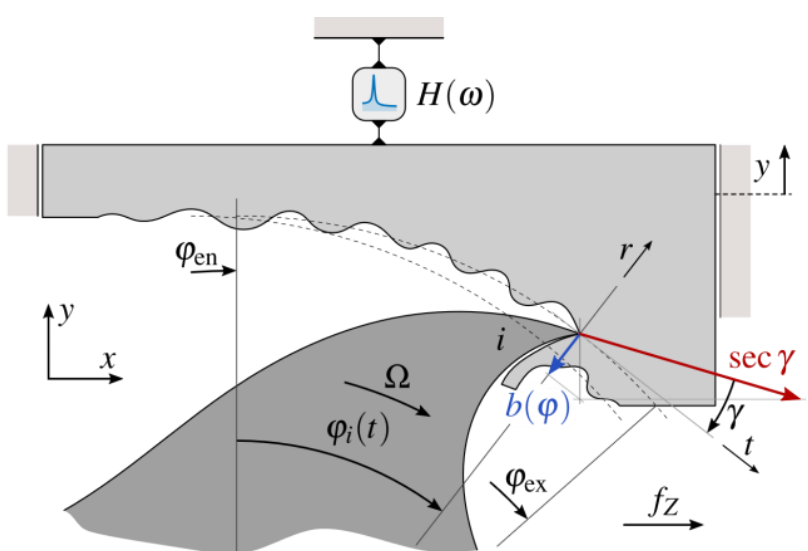

Fig. $1 y$-directional regenerative milling force model for thinwalled parts. Graphical obtention of the directional factor $b(\varphi)$.

delay is represented by $\tau$ and is equal to the tooth passing period $\square_{\square}=2 \pi /(\Omega \square)$ in this case. $\square(\square)=\square\left(\square+\square_{\square}\right)$ is the time-periodic directional factor, condensing the projection of the cutting force onto the vibration direction $(y)$ and, this, in turn, projected onto the chip thickness direction $(r)$.

\subsection{Formulation of the stability problem in the frequency domain}

The DDE in (1) is time-periodic due to the aforementioned time-periodicity of the directional factor, exhibiting both Hopf and period-doubling instabilities. Instead of using purely numerical time-discrete methods that could 'hide' the key relations between the machining parameters and stability properties, the stability of (1) can be studied in the frequency domain. To this effect, the critically stable case where the system vibrates at a dominant frequency $\omega_{\mathrm{c}}$ and its infinite $k$ modulations at the tooth passing frequency $\square_{\square}=2 \pi / T_{Z}$ is considered, that is,

$$
y(t)=\sum_{k=-\infty}^{\infty} y_{k} \mathrm{e}^{\mathrm{i}\left(\omega_{\mathrm{c}}+k \omega_{\mathrm{Z}}\right) t}
$$

$y(t-\tau)-y(t)=\left(\mathrm{e}^{-\mathrm{i} \omega_{\mathcal{c}} \tau}-1\right) \sum_{k=-\infty}^{\infty} y_{k} \mathrm{e}^{\mathrm{i}\left(\omega_{\mathrm{c}}+k \omega_{Z}\right) t}$.

In addition, the time-periodic directional factor $B(t)$ can be expressed as a Fourier series as

$B(t)=\sum_{k=-\infty}^{\infty} B_{k} \mathrm{e}^{\mathrm{i} k \omega_{Z} t}:=\frac{Z}{2 \pi} \sum_{k=-\infty}^{\infty} \beta_{k} \mathrm{e}^{\mathrm{i} k \omega_{Z} t}$

Each $k$ harmonic of both system displacement and force can be related in the frequency domain as $\square_{\square}=\square_{\square} \square_{\square}$, where $\square_{\square}=\square\left(\square_{\mathrm{c}}+\square \square_{\square}\right.$ ) is the receptance function evaluated at the $k$ modulated frequency and where multiple $y$-directional modes can be considered. Therefore, the characteristic equation of the $k$ harmonic results in

$y_{k}=\frac{a K_{t \mathrm{c}} Z}{2 \pi}\left(\mathrm{e}^{-\mathrm{i} \omega_{\mathrm{c}} \tau}-1\right) \sum_{r=-\infty}^{\infty} H_{k} \beta_{k-r} y_{r}$.

Hence, if all the $h$ harmonics are considered, the milling process stability analysis drives to the following infinite dimensional eigenvalue problem: 


\section{HSM \\ 2021}

MM Science Journal | www.mmscience.eu

ISSN 1803-1269 (Print) | ISSN 1805-0476 (Online)

Special Issue | HSM 2021

$16^{\text {th }}$ International Conference on High Speed Machining

October 26-27, 2021, Darmstadt, Germany

DOI: 10.17973/MMSJ.2021_11_2021167

$\left\{\begin{array}{c}y_{-h} \\ \vdots \\ y_{0} \\ \vdots \\ y_{h}\end{array}\right\}=\Lambda\left[\begin{array}{ccccc}H_{-h} & \ldots & 0 & \ldots & 0 \\ \vdots & \ddots & \vdots & \ddots & \vdots \\ 0 & \ldots & H_{0} & \ldots & 0 \\ \vdots & \ddots & \vdots & \ddots & \vdots \\ 0 & \ldots & 0 & \ldots & H_{h}\end{array}\right]\left[\begin{array}{ccccc}\beta_{0} & \ldots & \beta_{-h} & \ldots & \beta_{-2 h} \\ \vdots & \ddots & \vdots & \ddots & \vdots \\ \beta_{h} & \ldots & \beta_{-0} & \ldots & \beta_{-h} \\ \vdots & \ddots & \vdots & \ddots & \vdots \\ \beta_{2 h} & \ldots & \beta_{h} & \ldots & \beta_{0}\end{array}\right]\left\{\begin{array}{c}y_{-h} \\ \vdots \\ y_{0} \\ \vdots \\ y_{h}\end{array}\right\}$,

where

$\Lambda=\frac{a K_{t \mathrm{c}} Z}{2 \pi}\left(\mathrm{e}^{-\mathrm{i} \omega_{\mathrm{c}} \tau}-1\right)$

is the eigenvalue.

\subsection{Period-doubling stability}

Period-doubling instability takes place when the main harmonic $\left(0^{\text {th }}\right)$ and one of its $k^{\text {th }}$ modulations excite the same mode but at opposite sides of the ordinate axis, exhibiting a flip chatter frequency at $\square_{\mathrm{c}}=\square \omega_{\square} / 2$. Because $\left(\mathrm{e}^{-\mathrm{i} \square \mathrm{c}}-1\right)=0$ when an even harmonic is considered, only odd harmonics $(-1,-3,-5, \ldots)$ can generate the subsequent flip lobes. Nevertheless, the magnitude of the harmonics decrease as higher modulations are considered. Hence, flip lobes of order higher than 1 (main flip lobe) seldom limit the stability. Accordingly, the main flip lobe can be described by just considering the $0^{\text {th }}$ and the $-1^{\text {st }}$ harmonics in (5) [Zatarain 2010, Munoa 2013, Iglesias 2016], leading to the following $2 \times 2$ eigenvalue problem:

$\left\{\begin{array}{c}y_{-1} \\ y_{0}\end{array}\right\}=\Lambda\left[\begin{array}{cc}H_{-1} & 0 \\ 0 & H_{0}\end{array}\right]\left[\begin{array}{cc}\beta_{0} & \beta_{-1} \\ \beta_{1} & \beta_{0}\end{array}\right]\left\{\begin{array}{c}y_{-1} \\ y_{0}\end{array}\right\}$,

where $\beta_{0}$ is the mean directional factor and $\square_{-1}=\square_{1}^{*}$ is the $1^{\text {st }}$ harmonic of the directional factor. At the main flip lobe the flip chatter frequency is $\square_{\mathrm{c}}=\omega_{\square} / 2$, and thus $\mathrm{e}^{-\mathrm{i} \square_{\mathrm{c}} \square}$ $1=-2$, resulting in

$\operatorname{det}\left(\left[\begin{array}{ll}1 & 0 \\ 0 & 1\end{array}\right]+\frac{a K_{t c} Z}{\pi}\left[\begin{array}{cc}H_{-1} & 0 \\ 0 & H_{0}\end{array}\right]\left[\begin{array}{cc}\beta_{0} & \beta_{1}^{*} \\ \beta_{1} & \beta_{0}\end{array}\right]\right)=0$.

Due to the symmetry of the receptance function $\square_{-1}=$ $\square_{0} \mathrm{e}^{-\mathrm{i} 2 \square}$, being $\psi$ the phase of the receptance. Thus, the determinant can be reformulated as

$\operatorname{det}\left(\left[\begin{array}{ll}1 & 0 \\ 0 & 1\end{array}\right]+\frac{a K_{t \mathrm{c}} Z H_{0}}{\pi}\left[\begin{array}{cc}\mathrm{e}^{-\mathrm{i} 2 \psi} & 0 \\ 0 & 1\end{array}\right]\left[\begin{array}{cc}\beta_{0} & \beta_{1}^{*} \\ \beta_{1} & \beta_{0}\end{array}\right]\right)=0$.

Solving the determinant, the following closed-form expression of the flip chatter lobe can be devised

$a=-\frac{\pi\left(\cos \psi \pm \sqrt{r_{\beta}^{2}-\sin ^{2} \psi}\right)}{K_{t \mathrm{c}} Z \beta_{0}\left(1-r_{\beta}^{2}\right)\left|H_{0}\right|}$,

where $\square_{\square}=\left|\square_{1}\right| /\left|\square_{0}\right|$ is the ratio between the $1^{\text {st }}$ and the $0^{\text {th }}$ harmonics of the directional factor.

If the minimum —critical- point of the flip lobe is of interest, (10) can be expressed as a function of the dimensionless flip chatter frequency $\lambda=\omega_{\mathrm{c}} / \omega_{\mathrm{n}}=\square_{\square} /\left(2 \square_{\mathrm{n}}\right)$ as

$a(\lambda)=-\frac{\pi k\left[\left(1-\lambda^{2}\right) \pm \sqrt{r_{\beta}^{2}\left(1-\lambda^{2}\right)^{2}-4\left(1-r_{\beta}^{2}\right) \zeta^{2} \lambda^{2}}\right]}{K_{t \mathrm{c}} Z \beta_{0}\left(1-r_{\beta}^{2}\right)}$.

Then, for obtaining the minimum, (11) is derived with respect the dimensionless chatter frequency as follows:

$$
\frac{\partial}{\partial \lambda}\left(1-\lambda^{2}+\sqrt{r_{\beta}^{2}\left(1-\lambda^{2}\right)^{2}-4\left(1-r_{\beta}^{2}\right) \zeta^{2} \lambda^{2}}\right) \equiv 0,
$$

from which the dimensionless chatter frequency associated to the critical point is attained as:

$\lambda_{\min } \cong \begin{cases}\sqrt{1+\left(\frac{\zeta}{r_{\beta}}\right)^{2}}+\frac{\zeta}{r_{\beta}}, \quad \beta_{0}>0, \\ \sqrt{1+\left(\frac{\zeta}{r_{\beta}}\right)^{2}}-\frac{\zeta}{r_{\beta}}, \quad \beta_{0}<0 .\end{cases}$

Finally, by introducing the frequency of the minimum in (11), the critical depth of cut $a_{\min }$ of the period-doubling lobe

$a_{\mathrm{min}} \cong \frac{2 \pi k \zeta \lambda_{\min }}{K_{t \mathrm{c}} Z\left|\beta_{1}\right|}$

is achieved.

\section{ANALYSIS OF THE FLIP STABILITY THROUGH THE DIRECTIONAL FACTOR HARMONICS}

\subsection{Calculation of the directional factor in thin wall milling}

The $0^{\text {th }}$ and $1^{\text {st }}$ harmonics of the directional factor, $\beta_{0}$ and $\beta_{1}$, and the ratio between them $r_{\beta}$ have a direct influence in both shape and magnitude of the period-doubling stability limit. In thin wall milling, as the vibration direction is always the $y$ direction, the directional factor is predefined and independent of the system dynamics or other process parameters. Its value can be obtained by summing the contributions $b(\varphi)$ of the $Z$ cutting edge pondered through the screen function $g(\varphi)$ which equals 1 if the tooth is inside the engagement limits $\left[\varphi_{\mathrm{en}}, \varphi_{\mathrm{ex}}\right]$ and 0 if it is outside:

$B(t):=\sum_{i=1}^{Z} g\left(\varphi_{i}(t)\right) b\left(\varphi_{i}(t)\right)$,

where $\varphi_{\mathrm{i}}(t)$ stands for the angular position of the tooth $i$ at the instant $t$. The contribution of each tooth can be graphically obtained as in Fig. 1 by projecting the tangentially normalised cutting force onto the $y$ direction and this, in turn, projected onto the chip thickness direction $r$, which leads to

$b(\varphi)=\sec \gamma \sin (\gamma-\varphi) \cos \varphi=-\frac{1}{2} \sin 2 \varphi+\frac{k_{r}}{2}(1+\cos 2 \varphi)$

where $\square_{\square}:=\square_{\square \mathrm{c}} / \square_{\square \mathrm{c}}=\tan \square$ stands for the cutting force ratio. Thus, the $0^{\text {th }}$ (mean) directional factor $\beta_{0}$ and the $1^{\text {st }}$ modulation $\beta_{1}$ can be respectively obtained by performing the following integrals:

$\beta_{0}=\int_{\varphi_{\mathrm{en}}}^{\varphi_{\mathrm{ex}}} b(\varphi) \mathrm{d} \varphi=\frac{1}{4}\left[\cos 2 \varphi+2 k_{r} \varphi+k_{r} \sin 2 \varphi\right]_{\varphi_{\mathrm{en}}}^{\varphi_{\mathrm{ex}}}$

and

$\beta_{1}=\int_{\varphi_{\mathrm{en}}}^{\varphi_{\mathrm{ex}}} b(\varphi) \mathrm{e}^{\mathrm{i} Z \varphi} \mathrm{d} \varphi=$

$\left[\frac{-\mathrm{ie}^{\mathrm{i} Z \varphi}}{4}\left(\frac{2 k_{r}}{Z}+\frac{\mathrm{e}^{-\mathrm{i} 2 \varphi}\left(k_{r}-\mathrm{i}\right)}{Z-2}+\frac{\mathrm{e}^{\mathrm{i} 2 \varphi}\left(k_{r}+\mathrm{i}\right)}{Z+2}\right)\right]_{\varphi_{\mathrm{en}}}^{\varphi_{\mathrm{ex}}}$, 


\section{HSM \\ 2021}

MM Science Journal | www.mmscience.eu

ISSN 1803-1269 (Print) | ISSN 1805-0476 (Online)

Special Issue | HSM 2021

$16^{\text {th }}$ International Conference on High Speed Machining

October 26-27, 2021, Darmstadt, Germany

DOI: 10.17973/MMSJ.2021_11_2021167

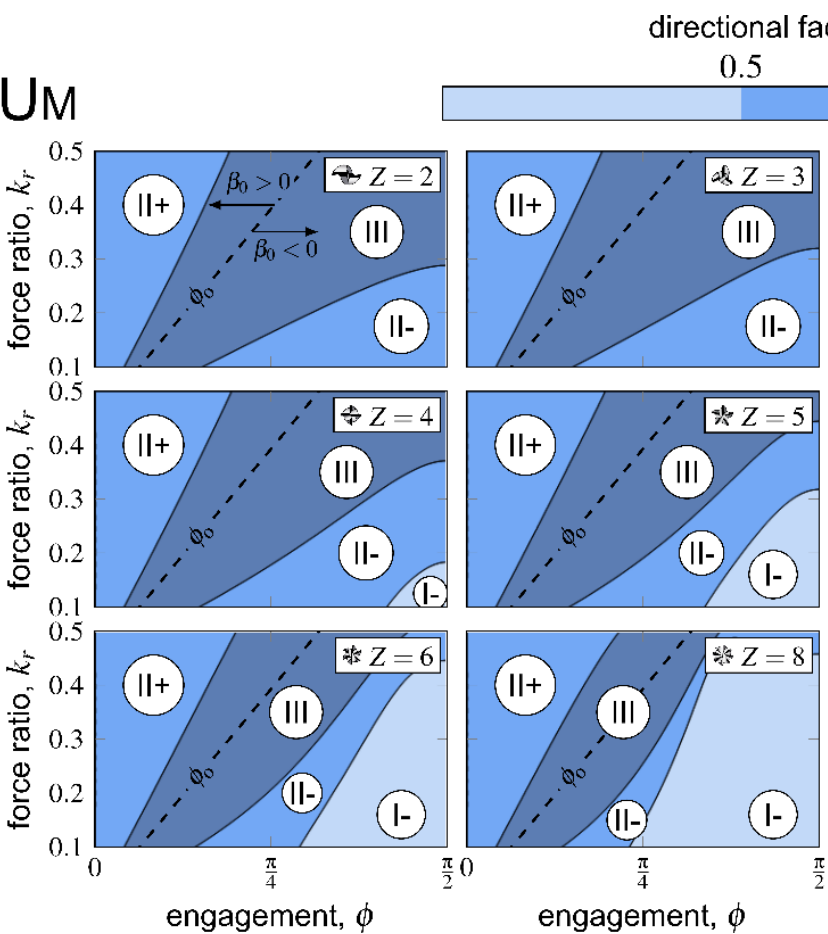

Fig. 2 Isocurves of the directional factor harmonic ratio number of teeth.

where the engagement limits $\left[\varphi_{\mathrm{en}}, \varphi_{\mathrm{ex}}\right]$ are defined through the engagement angle $\phi$ and the milling strategy $([0, \phi]$ for up-milling (UM) and $[\pi-\phi, \pi]$ for down-milling (DM)). However, immersions higher than the half-immersion case are hardly ever applied in thin-walled part milling as they may result in an excessive static deflection of the part.

Therefore, the $0^{\text {th }}$ and $1^{\text {st }}$ harmonics of the directional factor controlling the flip stability only depend on three dimensionless parameters: the combination of engagement angle $\phi$ and milling strategy, number of teeth $Z$ and the cutting force ratio $k_{r}$ (see Fig. 2). The latter is generally experimentally measured or estimated [Budak 1996], and oscillates within the range $0.1-0.5$ for most of the toolmaterial combinations. Thus, it is possible to study the influence of key process parameters, namely the radial engagement and number of teeth, on period-doubling stability in a dimensionless way by simply studying their effect on $\beta_{0}$ and $\beta_{1}$. This allows a more intuitive processplanning, as the optimisation of the process parameters based on the directional factor will remain optimal regardless of any variation of the part dynamics as a consequence of material removal during the machining process or along the tool axis as a consequence of the local modes of the part.

Based on this, the shape, relative location and magnitude of the flip stability limit is studied in the following sections.

\subsection{Shape and relative location of the flip lobe and Hopf/flip prevalence: cutting zones.}

The ratio between the first and zeroth harmonics of the directional factor $r_{\beta}$ is a measure of how interrupted the regeneration is. Accordingly, its value, together with the sign of $\beta_{0}$, control the shape, relative location and prevalence of the flip lobes [Iglesias 2016].

Hence, for thin-walled part milling we can study what kind of lobe distribution is expected depending on the engagement and the number of flutes, regardless of the system dynamics. Fig. 2 shows the $\phi-k_{r}$ isocurves of the harmonic ratio $r_{\beta}$ for the most common teeth configurations $(Z=2,3,4,5,6,8)$, for up- and down-milling strategies. According to these charts, it is possible to identify three cutting zones for thin wall milling:

- Zone I Continuous cutting zone. At this zone, $r_{\beta}<0.5$ : the cutting fluctuates very little with respect to the mean value, so Hopf minimum is below the flip minimum. This is achieved for 4 or more flutes and immersions nearby half-immersion. For UM, it will be a negative directional factor cutting ( I-,$\beta_{0}<0$ ), so the flip lobe will be located to the left of $\Omega=2 \square_{n} / Z$ and the flip chatter frequency will be below the natural frequency of the part. For DM, it will be a positive directional factor cutting, $\left(\mathbf{I}+, \beta_{0}>0\right)$ so it will be located to the right, with chatter frequencies above $\omega_{\mathrm{n}}$.

- Zone II Interrupted cutting zone. At this zone, $0.5<r_{\beta}$ $<1$ : the peak-to-peak oscillation of the cutting is larger to the mean value, so the flip minimum will dominate over Hopf. It will take place for cutters with 2 or 3 flutes or in the very low radial immersion zone for any number of teeth. The directional factor will mostly be positive (II+), so the flip lobe will be located to the right of $\Omega=$ $2 \square_{\mathrm{n}} / \mathrm{Z}$ most of the times. Negative directional factor cutting (II-) is however possible if few flutes and immersions higher than $\phi_{0}$ are used under UM.

- Zone III: Highly stable cutting zone. At this zone, $r_{\beta}>$ 1: the peak-to-peak value of the cutting is at least two times the mean value. The flip lobe will dominate and the vast lobe will be located at both sides of $\Omega=2 \square_{\mathrm{n}} / \mathrm{Z}$, 


\section{(C) HSM \\ 2021 \\ MM Science Journal | www.mmscience.eu \\ ISSN 1803-1269 (Print) | ISSN 1805-0476 (Online) \\ Special Issue | HSM 2021 \\ $16^{\text {th }}$ International Conference on High Speed Machining \\ October 26-27, 2021, Darmstadt, Germany}

DOI: 10.17973/MMSJ.2021_11_2021167

with chatter frequencies going from below to above of $\omega_{\text {n. }}$ It is only possible under UM and nearby $\phi_{0}$.

This may lead to the wrong conclusion that zone III presents a very large flip chatter likelihood because the fluctuation of the cutting is very large. Fig. 3 shows the evolution of $\beta_{0}, \beta_{1}$ and $r_{\beta}$ with the engagement for the $Z=3$ and $k_{r}=0.4$ case. As the engagement approaches $\phi_{0}$, the mean value of the directional factor decreases as the projection of the tangential force cancels the radial one dominating at very low immersions. Consequently, the period-doubling behaviour becomes dominant over the traditional Hopf even for a very low oscillatory cutting and hence a high flip critical stability. Because of that, zone III is the most preferable zone for achieving a stable cutting.

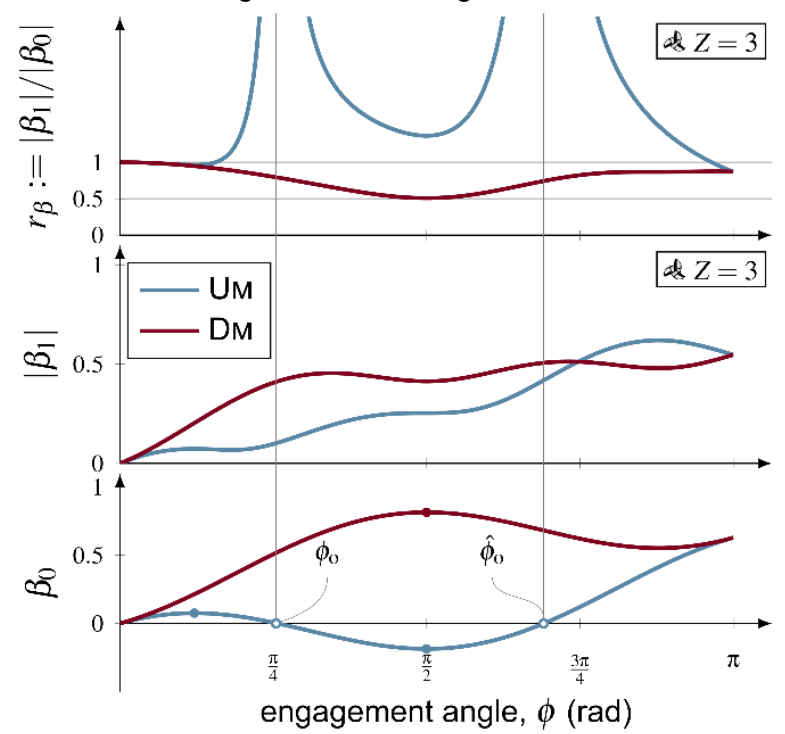

Fig. 3 Evolution of the mean directional factor $\beta_{0}$, amplitude of the first harmonic of the directional factor $\left|\beta_{1}\right|$ and the ratio $r_{\beta}$ for $k_{r}=0.4, Z=3$ and increasing radial engagement.

\subsection{Optimal engagement for flip bifurcation}

Once the shape and location of the flip lobe with the variation of the directional factor relative to its mean value $\left(r_{\beta}\right)$ are defined, the second key aspect is the absolute minimum depth of cut point of the lobe, provided by the absolute variation of the directional factor with respect to its mean value, related to $\left|\beta_{1}\right|$.

In thin-walled parts damping is usually very low and, consequently, the flip chatter frequency at the minimum is very close to $\omega_{\mathrm{n}}$, that is, $\lambda_{\min } \approx 1$. Consequently, the influence of process parameters in the flip stability limit on (14) is fully condensed in the amplitude of the first harmonic $\left|\beta_{1}\right|$ and the number of teeth $Z$. Bearing this in mind, it is possible to define a dimensionless critical depth of cut as $\alpha=\left(\left|\beta_{1}\right| Z\right)^{-1}$, which uniquely depends on the radial engagement, number of flutes and the cutting force ratio.

When the aim is to maximise the Hopf stability in thin-walled part milling, it is possible to find an engagement at which the mean directional factor $\beta_{0}$ is cancelled and a theoretical infinite stability can be achieved [Sanz-Calle, 2021]. Similarly, if the flip stability of the main lobe is to be maximised, the amplitude of the of the first harmonic $\left|\beta_{1}\right|$ should be minimised.

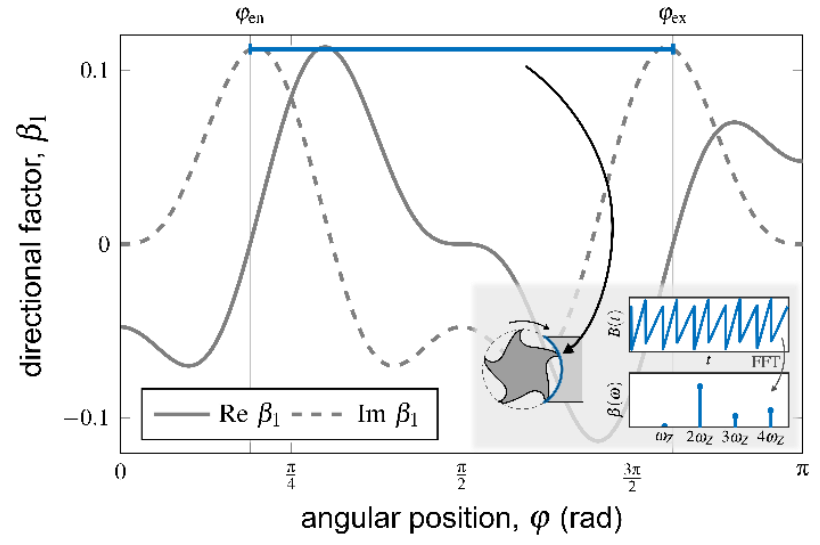

Fig. 4 Evolution of $\operatorname{Re} \beta_{1}$ and $\operatorname{Im} \beta_{1}$ with the angular position for $\mathrm{Z}=5$ and $k_{r}=0$ and the optimal case for $\left|\beta_{1}\right|=0$.

In this case, $\beta_{1}$ is a complex number, which implies that both real and imaginary parts of $\beta_{1}$ have to be cancelled for an ideal infinite stability. Unfortunately, cancelling $\operatorname{Re} \beta_{1}$ and $\operatorname{Im}$ $\beta_{1}$ at the same time for a fixed value of $k_{r}$ and $Z$ is only possible for certain pairs of $\varphi_{\mathrm{en}}$ and $\varphi_{\mathrm{ex}}$, which generally do not correspond to up- or down-milling configurations or which may even not be realisable - apart from the slotting configuration if an odd number of flutes higher than 2 is considered-.

Fig. 4 shows the evolution of $\operatorname{Re} \beta_{1}$ and $\operatorname{Im} \beta_{1}$ with the angular position for the $Z=5$ and $k_{r}=0$ case. As can be observed, if either up- or down-milling strategies are set, it is not possible to find an engagement $\phi$ which simultaneously cancels both real and imaginary parts. Instead, the infinite period-doubling stability is found at an intermediate case, whose application in thin-walled part milling is unfeasible. Therefore, in thin-walled part milling it is not possible to find an engagement that cancels $\left|\beta_{1}\right|$, the theoretically infinite milling stability can only be achieved through a proper cutter helix tuning instead of a radial engagement tuning [Zatarain 2006, 2010].

Nonetheless, in cases where the effect of the helix cannot be exploited to cancel the directional factor harmonics, it is possible to find engagements for $U_{M}$ which, while not leading to infinite stability, they do provide maximum stability as shown in the dimensionless depth of cut $\phi-k_{r}$ isocurves in Fig. 5. These stability extrema can be calculated by simply deriving $\left|\beta_{1}\right|^{2}$ with respect the engagement angle $\phi$ for the two milling configurations and setting it equal to zero, that is,

$$
\begin{aligned}
& \frac{\mathrm{d} \mid \beta_{1, \mathrm{UM}}^{\mathrm{UM}}}{\mathrm{d} \phi}=-\frac{2 \cos \phi\left(\mp \sin \phi+k_{r} \cos \phi\right)}{Z\left(Z^{2}-4\right)} \\
& \frac{\left( \pm Z(\cos 2 \phi-\cos Z \phi)+\left(Z \sin 2 \phi-\left(Z^{2}-2\right) \sin Z \phi\right) k_{r}\right)}{Z\left(Z^{2}-4\right)} \equiv 0 .
\end{aligned}
$$

An extremum will take place when any of the three factors in (19) becomes zero. In the first term, it is easy to see that the half-immersion $\phi=\pi / 2$ situation is an extremum for both UM and DM. The extremum provided by the second term, which is a practical value only under UM and which is independent of the number of flutes, results in 


\section{HSM \\ 2021}

MM Science Journal | www.mmscience.eu

ISSN 1803-1269 (Print) | ISSN 1805-0476 (Online)

Special Issue | HSM 2021

$16^{\text {th }}$ International Conference on High Speed Machining

October 26-27, 2021, Darmstadt, Germany

DOI: 10.17973/MMSJ.2021_11_2021167

UM
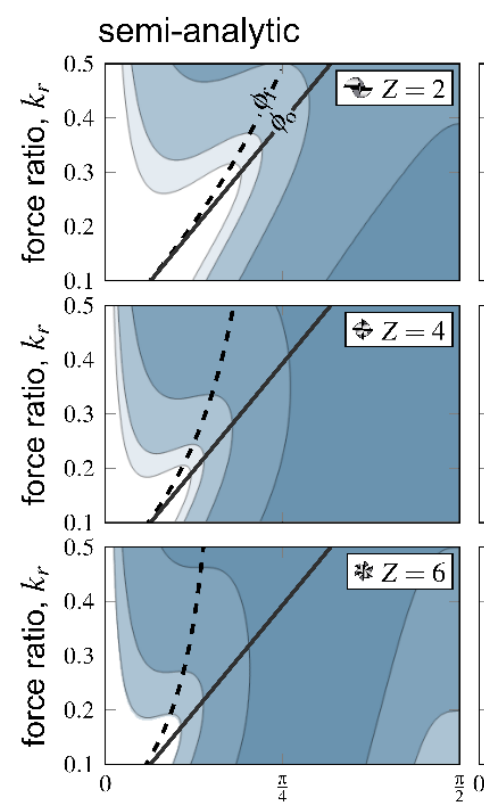

engagement, $\phi$ (rad)

dimensionless critical depth of cut, $\alpha$

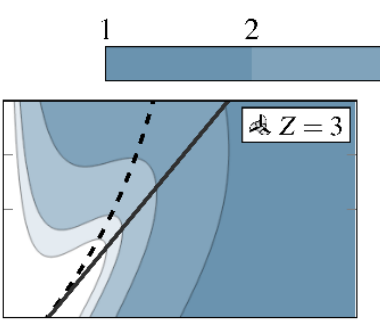

\begin{tabular}{lll}
5 & $10 \quad 15$ \\
\hline
\end{tabular}
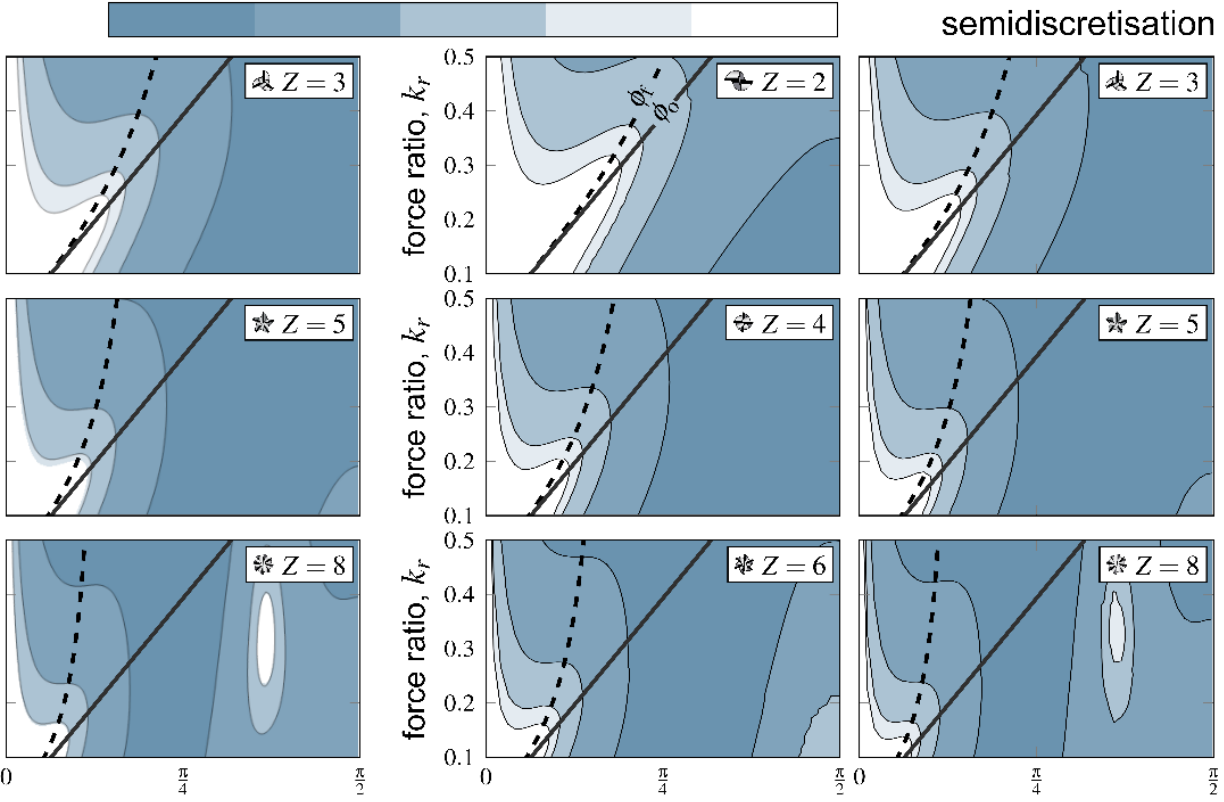

engagement, $\phi$ (rad)

engagement, $\phi$ (rad)

Fig. 5 Dimensionless depth of cut isocurves for up-milling and different number of flutes. Optimal engagements for flip and Hopf chatter are provided in dashed and continuous black lines, respectively. Left charts: isocurves obtained semianalytically. Right charts: isocurves obtained with semidiscretisation for the $\zeta=1.96 \%$ case from Tab. 1 .

$\left(-\sin \phi+k_{r} \cos \phi\right) \equiv 0 \quad \Rightarrow \quad \phi=\arctan k_{r}=\gamma$,

coinciding with the stability minimum of Hopf chatter [SanzCalle, 2021]. This engagement represents a local stability minimum unless cutters with $Z=6$ and $k_{r}>0.41$ or $Z=8$ and $k_{r}>0.3$ are considered.

The rest of the engagement extrema are originated by the third term of (19) for both UM and DM:

$$
Z(\cos 2 \phi-\cos Z \phi)+\left(Z \sin 2 \phi-\left(Z^{2}-2\right) \sin Z \phi\right) k_{r} \equiv 0 .
$$

Among the latter, it is particularly interesting the stability maximum of UM in the low immersion zone shown in Fig. 5, as the optimals corresponding to higher engagements would have the counterpart of an excessive static deflection and considerable Hopf chatter likelihood. Unfortunately, the transcendental nature of (21) impedes the obtention of this engagement in a closed-form. Instead, it is possible to approximate this low immersion maximum $\phi_{\mathrm{f}}$ for $U_{\mathrm{M}}$ by means of a $2^{\text {nd }}$ order Padé estimate at $\phi=0$ of (21), leading to

$\phi_{\mathrm{f}} \approx \frac{\sqrt{12 Z^{2} k_{r}^{2}+9}-3}{Z^{2} k_{r}}$.

The expression (22) provides a useful rule for process planning whenever period doubling chatter is the limiting problem and the smoothing effect of the helix is not enough for achieving a stable cut. In any case, if this flip optimal is compared to the Hopf optimal provided in [Sanz-Calle, 2021] as in Fig. 5, it is noticed that the two optimals are actually quite close to each other, which means that a compromise for obtaining a high flip and Hopf limits can be easily reached by tuning the engagement.

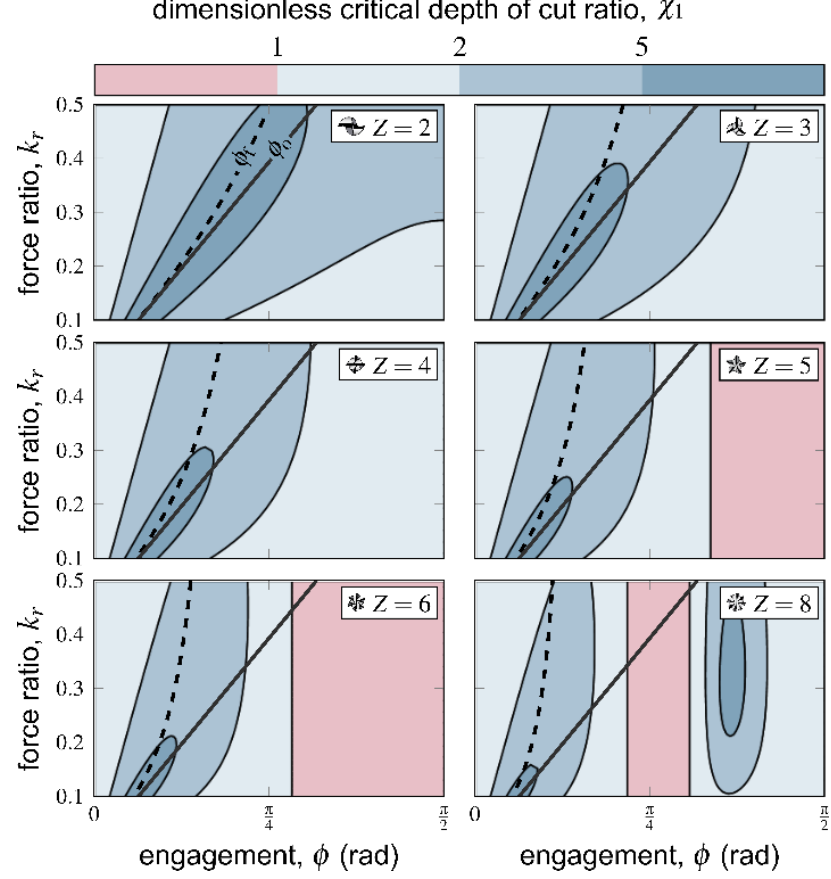

Fig. 6 Ratio of critical dimensionless depths of cut of the two milling configurations. Optimal engagements for flip and Hopf chatter are provided in dashed and continuous black lines, respectively.

\subsection{Comparison between milling strategies}

In order to compare the two milling directions in terms of absolute critical limit, the following ratio of dimensionless critical depths of cut is defined: 

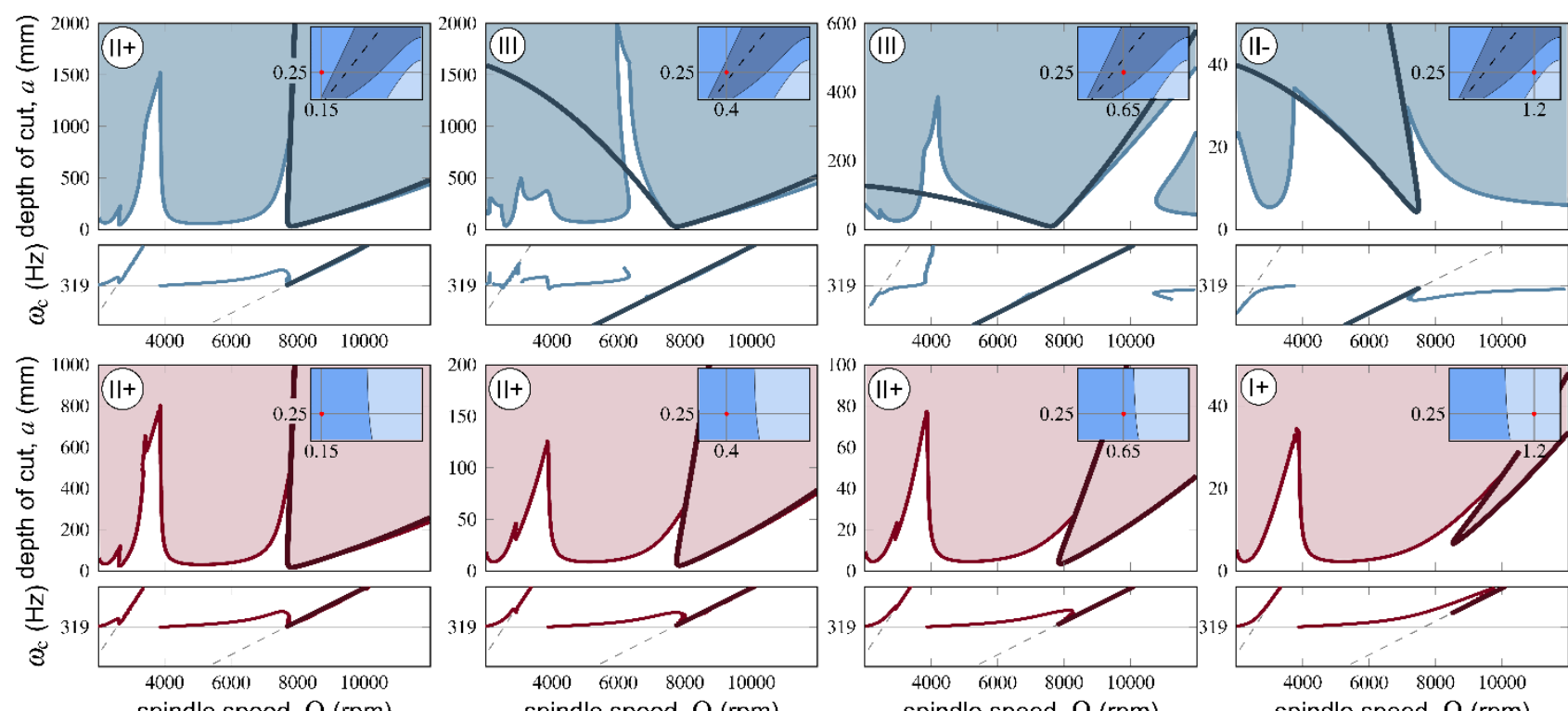

spindle speed, $\Omega$ (rpm)

spindle speed, $\Omega$ (rpm)

Fig. 7 Stability lobe diagrams for the $y$-directional milling case in Tab. 1 and $\phi=0.15,0.4,0.65$ and 1.2 rad engagement configurations. Upper charts: up-milling cases. Lower charts: down-milling cases. For a better insight, the analytical flip stability limit obtained from (11) is also provided in the stability charts.

$\chi_{1}:=\frac{\alpha_{\mathrm{UM}}}{\alpha_{\mathrm{DM}}}=\frac{\beta_{1, \mathrm{DM}}}{\beta_{1, \mathrm{UM}}}$

The $\phi-k_{r}$ isocurves of the ratio $\chi_{1}$ are depicted in Fig. 6 . Even though it has been previously seen that in UM wider flip chatter regions are expected due to their higher $r_{\beta}$, the absolute critical limit for flip chatter is generally higher for UM. With up to 4 flutes, UM will be superior regardless of the radial engagement. If more than 4 teeth are used, there exist engagement ranges within which DM presents a higher flip limit. Regardless of $k_{r}$, for $Z=5$ DM will be superior for immersions higher than $25.5 \%$, whereas for $Z=6$ DM will be superior for immersions higher than $18.3 \%$. If 8 flutes are considered, only immersions between $11.1 \%$ and $21.4 \%$ result in a higher limit for DM.

\section{VALIDATION ON MILLING CASES}

In this section, the aforementioned cutting zones and the optimal engagement situation formula are validated by means of semidiscretisation simulations [Insperger 2011] of the $y$-dominant milling case in Tab. 1 . In addition, the superior performance of up-milling strategy in terms of flip chatter is also validated.

The stability lobe diagrams for different engagement cases under up- and down- milling are depicted in Fig. 7. As predicted, for UM the aforementioned three period-doubling lobe shape configurations are possible. For very low immersion values, the cutting becomes very interrupted, the flip lobe is located to the right of $\Omega=2 \square_{n} / Z$, with chatter frequencies above $\omega_{\mathrm{n}}$. As the immersion increases and reaches the highly stable $r_{\beta}>1$ zone, lobes at both sides of $2 \square_{\mathrm{n}} / \mathrm{Z}$ take place regardless of the sign of $\beta_{0}$ and with chatter frequencies above and below $\omega_{\mathrm{n}}$. Finally, as the half immersion case is approached, the cutting is smoothed out and the flip lobe is located to the left of $2 \square_{n} / Z$, with chatter frequencies remain below $\omega_{\mathrm{n}}$. On the other hand, for downmilling, interrupted cutting takes place for low immersion cases. As half-immersion is approached, continuous cutting is achieved. In any case, a positive cutting always takes place under down-milling.

Tab. 1 Milling parameters with the $y$-directional dynamics from [Zatarain 2006] used for the validation.

\begin{tabular}{ccc}
\hline \multicolumn{3}{c}{ dynamics } \\
\hline$\omega_{\mathrm{n}}(\mathrm{Hz})$ & $\zeta(\%)$ & $k(\mathrm{~N} / \mu \mathrm{m})$ \\
\hline 319.375 & 1.96 & 21.7 \\
\hline \multicolumn{3}{c}{ tool $/$ process } \\
\hline$Z$ & $K_{\mathrm{tc}}(\mathrm{MPa})$ & $k_{r}$ \\
\hline 5 & 804.3 & 0.25 \\
\hline
\end{tabular}
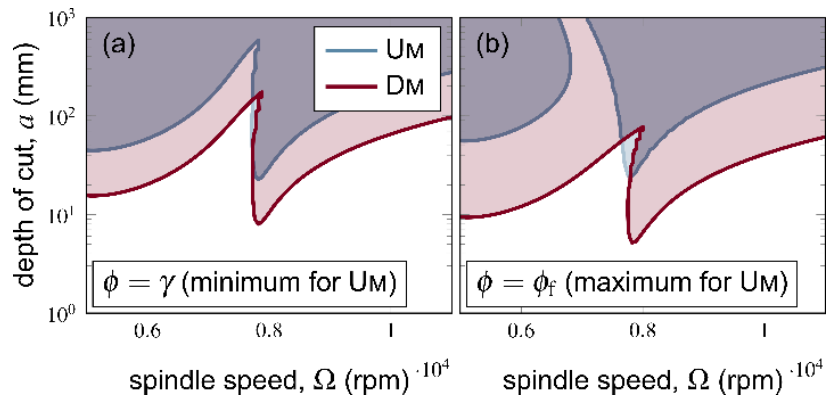

Fig. 8 Comparison of the flip stability limit of UM and $D M$ for two engagement cases. (a) $\phi=\gamma=0.245 \mathrm{rad}$ case. (b) $\phi=\phi_{\mathrm{f}}$ $=0.363 \mathrm{rad}$ case .

Regarding the absolute minimum depth of cut for flip chatter, Fig. 8 shows the comparison of the flip stability limit under up- and down-milling. Even for the local stability minimum $\phi=\gamma=0.245$ rad case, up-milling outperforms down-milling, showing a $187.5 \%$ higher minimum stability limit. Moreover, if the optimal engagement $\phi_{\mathrm{f}}=0.363 \mathrm{rad}$ provided in (22) is considered, this difference grows up to $400 \%$ in favour of up-milling. 


\section{HSM \\ 2021}

MM Science Journal | www.mmscience.eu

ISSN 1803-1269 (Print) | ISSN 1805-0476 (Online)

Special Issue | HSM 2021

$16^{\text {th }}$ International Conference on High Speed Machining

October 26-27, 2021, Darmstadt, Germany

DOI: 10.17973/MMSJ.2021_11_2021167

The here provided expressions and dimensionless charts have demonstrated that the flip lobe shape can be conveniently chosen an its critical depth of cut maximised by properly choosing the engagement conditions. By just experimentally measuring the cutting force ratio $k_{r}$, the optimal engagement for maximum flip stability can be determined from (22). After that, the $0^{\text {th }}$ and $1^{\text {st }}$ directional factor harmonics can be respectively calculated in (17) and (18), which can be used for determining the cutting zone in Fig. 2 and the critical depth of cut in (14).

Compared to Hopf-kind of chatter, the capacity of maximising the flip stability limit by tuning the radial engagement is however much more limited. Hence, beyond a certain point it may not be sufficient to tune the radial engagement to achieve a chatter-free machining. In these cases, stability can only be increased or even made infinite by properly adjusting the helix pitch to the target axial depth of cut [Zatarain 2006, 2010].

\section{CONCLUSIONS}

Period-doubling chatter can pose a real limitation to the productivity when milling thin-walled parts, as the low radial immersion and the few number of flutes much promote the interrupted cutting that gives rise to flip instability. The most widespread general stability algorithms such as semidiscretisation can handle this interrupted cutting, but their purely numerical nature turns the process planning task into a long taking trial and error process. However, in thin-walled part milling a dominant modal direction perpendicular to the feed motion is most likely to exist. This allows condensing the directional factor matrix onto a single coefficient, whose first and zeroth harmonic can be used for reformulating the stability problem in the frequency domain in a very simple form.

By these means, this paper studies the period-doubling stability of thin wall milling by inspecting the influence of key process parameters, namely, the number of flutes and radial engagement, on the mean and first directional factor harmonics. The dimensionless charts for predicting the flip lobe shape and Hopf/flip prevalence for both up- and downmilling are provided, which demonstrate the larger flip prevalence in the former. However, when it comes to achieve a higher absolute stability limit, up-milling outperforms down-milling under almost every practical engagement and teeth number combination. An expression for determining the optimal low-immersion engagement for a maximum stability under up-milling is also provided. However, as opposed to Hopf chatter case, it is demonstrated that it is not possible to totally cancel the period-doubling chatter by simply tuning the radial engagement, which indicates that the only feasible way to totally eliminate the flip chatter is tuning the cutter helix. Finally, the introduced findings are validated through semidiscretisation simulations.

\section{ACKNOWLEDGMENTS}

This research work has been done under the framework of the project MIRAGED (CER-20191001).

\section{REFERENCES}

[Altintas 1995] Altintas, Y., Budak, E. Analytical Prediction of Stability Lobes in Milling CIRP Annals, 1995, vol. 44, no. 1.

[Budak 1996] Budak, E., Altintas, Y., et al. Prediction of Milling Force Coefficients From Orthogonal Cutting Data Journal of Manufacturing Science and Engineering, 1996, vol. 118, no. 2 ISSN 10871357.

[Budak 1998] Budak, E., Altintas, Y. Analytical Prediction of Chatter Stability in Milling-Part I: General Formulation Journal of Dynamic Systems, Measurement and Control, Transactions of the ASME, 1998, vol. 120, no. 1 ISSN 15289028.

[Davies 2000] Davies, M. A., et al. Stability of Low Radial Immersion Milling CIRP Annals - Manufacturing Technology, 2000, vol. 49, no. 1 ISSN 00078506.

[Ding 2010] Ding, Y., et al. A Full-Discretization Method for Prediction of Milling Stability International Journal of Machine Tools and Manufacture, 2010, vol. 50, no. 5, ISSN 08906955.

[Iglesias 2016] Iglesias, A., et al. Analytical Expressions for Chatter Analysis in Milling Operations with One Dominant Mode Journal of Sound and Vibration, 2016, vol. 375, ISSN 10958568.

[Insperger 2011] Insperger, T., Stepan, G. SemiDiscretization for Time-Delay Systems 2011.

[Merdol 2004] Merdol, S.D., Altintas, Y. Multi Frequency Solution of Chatter Stability for Low Immersion Milling Journal of Manufacturing Science and Engineering, Transactions of the ASME, 2004, vol. 126, no. 3 ISSN 10871357.

[Minis 1993] Minis, I., Yanushevsky, R. A New Theoretical Approach for the Prediction of Machine Tool Chatter in Milling Journal of Manufacturing Science and Engineering, Transactions of the ASME, 1993, vol. 115, no. 1 ISSN 15288935.

[Munoa 2016] Munoa, J., et al. Chatter Suppression Techniques in Metal Cutting CIRP Annals - Manufacturing Technology, 2016, vol. 65, no. 2.

[Munoa 2013] Munoa, J., et al. Interaction between Multiple Modes in Milling Processes Machining Science and Technology, 2013, vol. 17, no. 2 ISSN 10910344.

[Sanz-Calle 2021] Sanz-Calle, M., et al. The influence of radial engagement and milling direction for thin wall machining: a semi-analytical study. Procedia CIRP, 2021.

[Tlusty 1963] Tlusty, J., Polacek, M. The Stability of Machine Tools against Self-Excited Vibrations in Machining Proceedings of the ASME International, 1963.

[Tobias 1958] Tobias, S. A., Fishwick, W. Theory of Regenerative Machine Tool Chatter The Engineer, 1958.

[Zatarain 2006] Zatarain, M., et al. Analysis of the Influence of Mill Helix Angle on Chatter Stability CIRP Annals Manufacturing Technology, 2006, vol. 55, no. 1 ISSN 00078506.

[Zatarain 2010] Zatarain, M., et al. Analysis of Directional Factors in Milling: Importance of Multi-Frequency Calculation and of the Inclusion of the Effect of the Helix Angle International Journal of Advanced Manufacturing Technology, 2010, vol. 47, no. 5-8 ISSN 02683768. 